\title{
Outbreak of Gastroenteritis due to Salmonella virchow in a Maternity Hospital
}

\author{
BERNARD ROWE,* M.A., M.B., D.T.M.\&H. ; CHRISTOPHER GILES, $\dagger$ M.D., F.C.PATH. \\ G. LAING BROWN $\ddagger$ M.D., D.P.H.
}

British Medical fournal, 1969, 3, 561-564

\begin{abstract}
Summary : An outbreak of gastroenteritis due to Salmonella virchow occurred in a maternity hospital in the Midlands in October-November 1968. Twenty-six babies and six mothers were infected. Fifteen of the babies had diarrhoea and $\mathbf{1 0}$ of these were in the special care baby unit. The only fatal case was that of an infant with gross congenital malformations.

The outbreak followed the admission of a patient to the delivery suite who was subsequently shown to be a symptomless excreter of $\mathbf{S}$. virchow. A major factor in the spread of this organism is thought to have been heavy environmental contamination resulting from diarrhoea due to infection of her newborn baby.
\end{abstract}

\section{Introduction}

Organisms of the salmonella group are often found in outbreaks of gastroenteritis in hospitals. In the 10-year period 1958-67 409 outbreaks of "food-poisoning" were reported from hospitals in England and Wales. In 249 (61\%) of these outbreaks the cause was shown to be a salmonella gastroenteritis (Report, 1959, 1960, 1961, 1962, 1963, 1964 ; Vernon, 1965, 1966, 1967, 1969).

Previous to 1967 there had been few human isolations of Salmonella virchow in England and Wales. The reconds of the Epidemiological Research Laboratory of the Public Health Laboratory Service show that this serotype was isolated from 107 cases or symptomless excreters in the five years 1962-6an annual average of 21 isolations. In 1967 and 1968, respectively, 234 and 476 human isolations were recorded. It is noteworthy that most of these infections occurred in the Midlands, though Semple, Turner, and Lowry (1968) described a large outbreak in the Liverpool area.

\section{Outbreak}

During the second and third weeks in October 1968 it was found that a number of babies were suffering from diarrhoea (see Table). From their faeces $S$. virchow was isolated, and the hospital was alerted to the fact that an outbreak due to this organism was in progress.

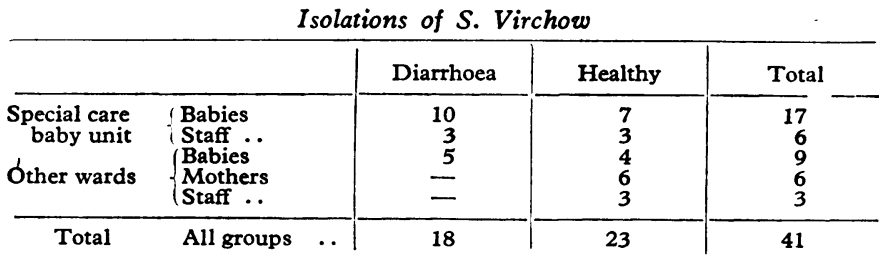

The hospital is the major maternity unit for a population of almost half a million, and was first opened in April 1968. The monthly average number of women delivered was 431 .

\footnotetext{
* Assistant Bacteriologist, Salmonella Reference Laboratory, Central Public Health Laboratory, London N.W.9.

† Consultant Pathologist, City General Hospital, Stoke-on-Trent, Staffs.

$\neq$ Consultant Physician in Infectious Diseases, Bucknall Hospital, Stoke-
} on-Trent, Staffs.
The accommodation in use at the time of the outbreak comprised a special care baby unit on the ground floor, one antenatal ward, and three postnatal wards on successive floors. Each ward comprised 28 beds for mothers and each postnatal ward had two nurseries.

The special care baby unit contained two single cubicles and five large rooms. Three of these large rooms had spaces for six cots or incubators, and the other two had spaces for seven each. The rooms were maintained at different conditions of humidity and temperature, babies being moved from room to room as their medical condition warranted. During the period of the outbreak the number of babies in the unit varied from 14 to 21 , with a daily average of 17 .

Mrs. X was admitted on 13 October and delivered some hours later on 14 October. Her baby was premature, and was taken direct to one of the large rooms in the special care baby unit. The mother was taken to the postnatal ward on the fourth floor.

\section{Special Care Baby Unit}

On 15 October Baby $\mathrm{X}$ was noticed to have diarrhoea, and was transferred to one of the cubicles in the special care baby unit. As no bacterial pathogens were found on culture of a stool specimen the baby was returned on 20 October to a large room, where he remained.

On 18 October three more babies in the special care baby unit had diarrhoea. All three had been in the same large room with Baby X, and stool cultures from them were reported to be negative for salmonella organisms. The fifth case of diarrhoea was reported on the 20th and a stool culture was again negative.

On 22 October $S$. virchow was found in a repeat stool specimen from one of the babies, and this serotype was subsequently isolated from Baby $\mathrm{X}$ and the other two diarrhoea cases. On 24 October one baby with exomphalos developed diarrhoea and died. At necropsy $S$. virchow was isolated. Two further cases of diarrhoea occurred on 24 October and three more on the 25 th, $S$. virchow being isolated from their faeces.

Stool cultures were examined from the remaining 11 babies in the special care baby unit and seven were found to be symptomless excreters of $S$. virchow (see Table).

At this stage admissions to the unit ceased and all babies were moved to an unused isolation unit in the hospital. The four uninfected babies were nursed in a separate room, but between 1 and 6 November three became symptomless excreters.

Ten more babies were at risk in the special care baby unit during the period following the admission of Baby $\mathrm{X}$. Three of these died from other recognized causes and seven were discharged before the outbreak was recognized. Stool cultures had not therefore been carried out on these babies.

\section{Other Wards}

When the nature of the infection was recognized faecal specimens were examined from all other patients and members of the staff. 
First Floor.-In this antenatal ward 30 mothers were at risk, but diarrhoea was not reported and no isolations of $S$. virchow were made.

Second Floor.-Eighty-four mothers were at risk. One symptomless excreter of $S$. virchow was found ; her baby had been infected in the special care baby unit and had been moved up to this floor before the infection was recognized. No other babies became infected on this floor.

Third Floor.-Seventy-five mothers and their babies were at risk; one mother and three babies were symptomless excreters of $S$. virchow.

Fourth Floor.-During the outbreak period 101 mothers and their babies had been accommodated. Four mothers were found to be symptomless excreters, one being the mother of Baby $\mathrm{X}$. Five babies developed diarrhoea, and $S$. virchow was isolated from the stools in all cases.

\section{Hospital Staff}

Three nurses working in the special care baby unit reported with diarrhoea, and $S$. virchow was isolated from their stools. Six symptomless excreters were found among the rest of the staff. Five of these were nurses; three had been employed in the special care baby unit, one in the delivery suite, and one had worked on floors 4 and 3 in succession. The sixth symptomless excreter was a porter who had worked on the delivery suite.

\section{Spread of Infection in the Hospital}

Mrs. X admitted to an attack of diarrhoea nine weeks before her admission to the maternity hospital. Since $S$. virchow was subsequently isolated from her stools she must be regarded as a symptomless excreter and the source of the infection in the hospital. Her baby was most probably infected at birth and subsequently started the chain of infection in the special care baby unit. Baby $\mathbf{X}$ was accommodated in several rooms in the unit and further cases of diarrhoea occurred in babies in close contact. Members of the nursing staff were infected at this time.

The mother on the second floor who was found to be a symptomless excreter acquired the organism from her baby, who had been in contact with Baby $\mathrm{X}$ in the special care baby unit before transfer to the second floor. Mrs. X was sent to the fourth floor after delivery, and $S$. virchow was isolated from mothers and babies in contact with her. Mrs. $X$ is known to have handled babies other than her own while in the ward. Three babies and one mother were infected on the third floor. A nurse who was subsequently shown to be infected had worked on the fourth floor in contact with Mrs. X before being transferred to the third floor.

\section{Source of Infection}

In June and July $1968 S$. virchow was isolated from eight cases of gastroenteritis in the area, and at that time a chain of infection was established between these cases and a shop supplying spit-roasted chickens in the same area. It was known that this shop and others in the area had received frozen chickens from a packing station in Cheshire which had been shown to be the source of the infected chickens responsible for an outbreak of gastroenteritis due to $S$. virchow in the Liverpool area in July 1968 (Semple et al., 1968 ; Pennington et al., 1968). During her pregnancy Mrs. X had been fond of roast chickens. These were obtained from a shop selling spit-roasted chickens cooked on the premises. This shop had received frozen chickens from the packing station in Cheshire.

\section{Control Measures}

As soon as the nature of the outbreak was apparent all admissions to the maternity hospital ceased and arrangements were made for the examination of stool specimens from all patients and staff. Three specimens were examined from all mothers, babies, nurses, doctors, porters, and all engineers and administrative staff. Those who were found to be infected with $S$. virchow were suspended from duty. All mothers and babies found to be positive were transferred to the local isolation hospital as soon as possible. On discharge mothers and babies known to be positive were notified to their local medical officer of health. Those who had been in contact with the infection in the hospital but discharged before the identification of the cause of the outbreak were also notified for investigation.

As soon as all patients who had been at risk were discharged the hospital was closed and terminal disinfection was begun. Each empty ward was washed down and all walls, ceilings, and floors were sprayed three times with a $1 \%$ solution of Tego (a bactericidal ampholytic surface-active agent). Bedding was steam-sterilized at a pressure of $20 \mathrm{lb}$./sq. in. $(1.406 \mathrm{~kg} . / \mathrm{sq}$. $\mathrm{cm}$.) and all non-disposable articles were washed in Tego. Small inexpensive items used in washing-up and cleaning were destroyed. Incubators were fumigated with formaldehyde and ammonia, and some electrical equipment was sterilized with ethylene dioxide gas.

\section{Discussion}

When a single article of food is contaminated with salmonella organisms and eaten by a large number of persons an explosive outbreak of gastroenteritis may occur. This is a familiar type of food-poisoning outbreak and its essential feature is the appearance of a large number of cases over a short period of time, usually about two days (Garrod and McIlroy, 1949). In many hospital outbreaks this explosive characteristic is absent and the epidemiological picture is that of cases occurring over a variable period of time (Taylor, 1963). Some outbreaks have continued for many months, which suggests a person-to-person spread (Bate and James, 1958). The term "food-poisoning" is hardly appropriate for this type of outbreak, and the use of the term is misleading in these circumstances.

This type of outbreak is not uncommon in maternity units, and usually follows the admission of a patient who may or may not have diarrhoea but whose stools contain salmonella organisms. She infects her baby at birth; a few days later that baby develops diarrhoea and then further cases of diarrhoea occur among the other babies. The source of infection is the mother, but the spread is usually from her baby with diarrhoea. The organism spreads by cross-infection, and multiplication in food plays no part in this.

\section{An Important Factor}

Parker (1954) reviewed the subject and emphasized "environmental contamination" derived from soiled napkins and bedding as an important factor in the spread of infection in wards where babies are nursed. Environmental contamination implies surface contamination around beds, contamination of other surfaces and objects, and spread by dust. Adults who pass formed but infected stools present little risk of environmental contamination, though they may be important sources of spread of infection in food-handling. Adults with diarrhoea are important sources of environmental contamination, as are babies passing infected fluid stools who may or may not show other clinical symptoms of salmonellosis. It is the state of the stool which determines the degree of environmental contamination. In salmonella outbreaks in baby units emphasis has previously been placed on the spread of infection by the attendant's hands, which had become contaminated during napkinchanging. Regular hand-washing and the provision of separate staff for napkin-changing and feeding must still remain essential nursing procedures, but sufficient evidence has now been produced to suggest that environmental contamination may be as important in many outbreaks. 
In the present outbreak, with so many cases of diarrhoea in young babies in one unit, the probability of heavy environmental contamination was great and it is likely this was a major factor in the spread of the organism throughout the special care baby unit. The rooms accommodated up to seven babies, and the movement of babies from room to room during the outbreak assisted in disseminating the organism. Unfortunately the investigation was retrospective and bacteriological proof of this could not be obtained.

The special care baby unit admitted babies who were either premature or had conditions other than gastroenteritis. It is well recognized that such babies form a highly susceptible group. Infection is dependent on the relation between host and parasite. In the special care baby unit the host was highly susceptible, and this helps to explain the apparent high infectivity of the organism which infected 16 out of the 17 babies at risk.

During the period of the outbreak about 250 mothers and their babies were at risk in the postnatal wards, and though the infection was introduced to each ward the spread within the wards was minimal. This supports Parker's contention that the infection seldom becomes established in wards unless most of the patients are under 2 years of age. In the three lying-in wards each baby, so far as was possible, was nursed with its mother in the ward and was cared for by the mother. The communal nursery was a necessity in the special care baby unit for practical considerations, and the majority of the babies became infected, whereas in the postnatal wards very few babies were infected, even though the infection was introduced. The need to avoid communal nurseries when at all possible is clearly demonstrated.

In this outbreak it is highly unlikely that the contamination of food, and the ensuing multiplication of the salmonella organisms, played any part in the spread within the hospital. The milk feeds for the babies in the special care baby unit and all wards were prepared in a central milk kitchen: "in-bottle" sterilization was carried out ; the entire unit of the bottle containing the feed with the teat in position and covered by a protective cap was subjected to heat sterilization. The feeds were then distributed to the wands and refrigerated until required. S. virchow was not isolated from dried milk powder, prepared milk feeds, or staff in the central milk kitchen. Had the milk feeds been infected an explosive outbreak involving babies on all floors could have been expected. The mothers in the maternity hospital received their meals from a kitchen which prepared meals for 700 patients in the general hospital. No isolations of $S$. virchow were made other than from patients in the maternity hospital. The ratio of cases to symptomless excreters among those infected with $S$. virchow in the hospital varied from group to group. Of the infected babies $60 \%$ had diarrhoea, while for the staff and mothers the proportion with diarrhoea was $33 \%$ and $0 \%$ respectively. The morbidity among infected staff was different from that described by Parker (1954), who stated that infected staff rarely developed symptoms. The high morbidity in babies compared with the much lower morbidity in mothers has been noted in previous outbreaks (Murray and Walker, 1958).

\section{Continued Excretion of Organisms}

After the return home of mothers and babies who were still excreting $S$. virchow in their stools, some family contacts became infected. At least 16 persons were known to have become infected in this way but only four of these had diarrhoea. This supports Parker's contention that within the family unit small amounts of organisms are transmitted which tend to produce symptomless excreters rather than cases of gastroenteritis.

Many of the babies in this outbreak were still excreting $S$. virchow several months later. Babies under 6 months old tend to excrete salmonella organisms for longer periods than older babies or adults. Rubenstein, Feemster, and Smith (1944) reported that $48 \%$ of infants under 6 months old who were infected with $S$. typhimurium still had positive stool cultures at 14 weeks. If such infants are under 6 weeks old they usually continue to excrete until they are about 1 year old and taking an adult type of diet (Taylor, 1965). It may be more harmful to the ultimate well-being of such a baby to be kept in hospital, and provided the family is able to practise careful hygiene the baby is best sent home.

In the case of adults, long-continued excretion of salmonella organisms may also occur. Of the infected nurses, five out of nine remained intermittent excreters for several months. Kwantes (1952) reported that $14 \%$ of the cases in an outbreak of S. typhimurium were still excreting the organism four weeks after the disappearance of clinical symptoms and that at least $2 \%$ were still excreting at nine weeks. Antibiotic treatment does not shorten the period of symptomless excretion of salmonella organisms, and in fact controlled studies suggest that the period may be lengthened (Dixon, 1965).

\section{Recommendations}

All hospitals face the danger of admitting the undiagnosed case of salmonella gastroenteritis or the symptomless excreter. On admission every patient must be questioned about recent attacks of diarrhoea and contact with other cases. A patient with a suggestive history must be isolated until bacteriological investigations have been completed. Should the patient be a maternity case delivered during this period her baby must also be isolated and investigated.

All patients with diarrhoea must be regarded as infectious and should be isolated until their faeces have been shown to be free from known pathogenic organisms. Failure to isolate pathogens from a single stool culture is not sufficient proof that the diarrhoea is non-infectious.

In a unit such as a special care baby unit the use of large rooms containing several babies may be necessary, but it must be recognized that this carries a high risk of cross-infection which can be minimized by restricting the transfer of babies from room to room. In a normal postnatal ward the avoidance of communal nurseries has been shown to reduce the risk of cross-infection (Medical Research Council, 1951).

All hospital patients are liable to cross-infection, but in units accommodating young babies the risk is greatest, because not only are they more susceptible than older children and adults but they are also more likely to spread infection, since they produce fluid stools. The first-line defence depends on prompt detection and isolation of the infected patient, which inevitably will fail on occasion, and the spread of infection can then be controlled only by efficient nursing. The duties of medical and nursing staff are clear in their role to combat crossinfection. The efficient execution of these duties requires that adequate isolation accommodation is readily available and that a sufficient number of trained nursing staff are on duty not only by day but also at night, so that careful attention to technique is not reduced (Medical Research Council, 1951).

\section{Postscript}

Since the epidemic all women with diarrhoea or a recent history of diarrhoea have been admitted to and delivered in the isolation unit of the hospital. During the first five months of this regimen more than $10 \%$ of these patients yielded positive specimens ; altogether six cases of previously undiagnosed salmonellosis and one of Sonne dysentery were discovered in this group.

Specimens of faeces from every member of the staff of the isolation unit have been examined at weekly intervals, but in spite of this precaution one intermittent carrier of $S$. virchow 
escaped detection and infected three infants, two of whom developed diarrhoea. The infection was, however, contained within the isolation unit.

We wish to acknowledge the help received from Dr. W. L. Hooper, Dr. C. R. Knappett, Dr. J. A. H. Brown, Dr. J. S. Hamilton, Dr. J. Warrack, Dr. D. C. Thursby-Pelham, and Dr. J. G. Dathan.

\section{REFERENCES}

Bate, J. G., and James, U. (1958). Lancet, 2, 713.

Dixon, J. M. S. (1965). British Medical fournal, 2, 1343.

Garrod, L. P., and McIlroy, M. B. (1949). British Medical fournal, 2, 1259.

Kwantes, W. (1952). Monthly Bulletin of the Ministry of Health and the Public Health Laboratory Service, 11, 239.

Medical Research Council (1951). Control of Cross-infection in Hospitals, pp. 5, 6, 38. London, H.M.S.O.

Murray, J. O., and Walker, J. H. C. (1958). Medical Officer, 100, 221. Parker, M. T. (1954). Royal Sanitary Institute fournal, 74, 847.

Pennington, J. H., Brooksbank, N. H., Poole, P. M., and Seymour, F. (1968). British Medical fournal, 4, 804.
Report (1959). Monthly Bulletin of the Ministry of Health and the Public Health Laboratory Service, 18, 169.

Report (1960). Monthly Bulletin of the Ministry of Health and the Public Health Laboratory Service, 19, 224.

Report (1961). Monthly Bulletin of the Ministry of Health and the Public Health Laboratory Service, 20, 160.

Report (1962). Monthly Bulletin of the Ministry of Health and the Public Health Laboratory Service, 21, 180

Report (1963). Monthly Bulletin of the Ministry of Health and the Public Health Laboratory Service, 22, 200.

Report (1964). Monthly Bulletin of the Ministry of Health and the Public Health Laboratory Service, 23, 189.

Rubenstein, A. D., Feemster, R. F., and Smith, H. M. (1944). American fournal of Public Health and the Nation's Health, 34, 841.

Semple, A. B., Turner, G. C., and Lowry, D. M. O. (1968). British Medical fournal, 4, 801.

Taylor, J. (1963). In Infection in Hospitals: Epidemiology and Control, edited by R. E. O. Williams and R. A. Shooter, p. 145. Oxford, Blackwell.

Taylor, J. (1965). Practitioner, 195, 12.

Vernon, E. (1965). Monthly Bulletin of the Ministry of Health and the Public Health Laboratory Service, 24, 321.

Vernon, E. (1966). Monthly Bulletin of the Ministry of Health and the Public Health Laboratory Service, 25, 194.

Vernon, E. (1967). Monthly Bulletin of the Ministry of Health and the Public Health Laboratory Service, 26, 235.

Vernon, E. (1969). Public Health (London), 83, 205.

\title{
Severe Depressive Mood Changes Following Slow-release Intramuscular Fluphenazine Injection
}

\author{
R. DE ALARCON,* M.D., D.P.M. ; M. W. P. CARNEY, † M.D., M.R.C.P.I., D.P.M.
}

\begin{abstract}
Gummary : Sixteen patients in whom schizophrenia was initially diagnosed and who were treated with fluphenazine enanthate or decanoate developed severe depression for a short period after the injection. In five cases this depression is thought to have been responsible for suicide. In 8 out of 10 cases the depression responded to electroplexy (E.C.T.). It is recommended that patients who are treated with fluphenazine should be carefully supervised.
\end{abstract}

\section{Introduction}

Slow-release intramuscular fluphenazine, both in the enanthate (Moditen) and in the decanoate (Modecate) form, is increasingly being used in the community treatment of schizophrenia on account of the simplicity of its administrationone injection every two to four weeks-and the assurance it gives that the patient has taken the medication in the dosage prescribed. In the past many chronic schizophrenics who responded to maintenance phenothiazine treatment thwarted efforts to rehabilitate them in the community by neglecting to take their medication orally in the amounts and at the time required. With fluphenazine enanthate many of these patients can now be maintained out of hospital (Bethell et al., 1966 ; Freeman, 1967). Nevertheless, if injected slow-release drugs are to mean a major advance in psychiatric treatment more attention must be paid to their side-effects as well as to their effects. Lack of knowledge of the precautions with which a drug should be administered can lead to its unwarranted total rejection by doctors if fatal or other adverse effects occur.

This paper describes depressive mood changes which may follow a slow-release injection of fluphenazine. The case

\footnotetext{
* Consultant Psychiatrist, Medical Research Council Clinical Psychiatry Unit, Graylingwell Hospital, Chichester.

t Consultant Psychiatrist, Blackpool and Fylde Hospital Group, and Lancaster Moor Hospital.
}

of one patient on whom a trial was conducted is reported in detail and 15 others are discussed, including five who committed suicide.

\section{Trial Case}

A 39-year-old unmarried man with a five-year history of mental illness was admitted to a mental hospital via a general hospital casualty department in November 1968 after he had attempted suicide by slashing his wrists.

Personal and Family History.-There was no family history of mental illness apart from one maternal uncle who had a "breakdown" after the first world war. Birth, early milestones, and schooling were normal. He was regarded as shy and "highly strung." At age 16 he became articled to a firm of accountants, qualifying as a chartered accountant at the age of 28 . He had worked in this capacity ever since.

Previous Psychiatric History.-In 1963 (aged 34) he began to complain that advertisements in The Times were being directed against him and that people were accusing him of being a Communist. The following year these ideas of reference became more pronounced, other typical schizophrenic symptoms appeared, and eventually he had to be admitted to a mental hospital under Section 25 on account of disturbed behaviour. The diagnosis made on this first admission was schizophrenia, and he was treated with chlorpromazine. He was readmitted-never for longer than five weeks-in 1965, 1966, and 1967 with the same diagnosis, and with similar symptomatology. On the last occasion, however, which occurred shortly after his father's death, he was given six treatments with E,C.T. On discharge in March 1967 intramuscular fluphenazine enanthate was prescribed on the grounds that he would not persevere with oral medication. He later moved to East London and arrangements were made with his Sussex general practitioner to give him a fortnightly injection of $25 \mathrm{mg}$. of fluphenazine enanthate when he came down to visit his mother. No psychiatric supervision was provided.-

Present Episode.-For three to four months before admission he had been having recurring bouts of severe depression about every fortnight. The usual pattern was to feel tired and sad on a Sunday evening and to wake up the next morning very depressed and low. 